\section{Venetoclax penetrates in cerebrospinal fluid and may be effective in chronic lymphocytic leukemia with central nervous system involvement.}

Venetoclax, a selective inhibitor of BCL2, has shown promising efficacy in chronic lymphocytic leukemia (CLL) both as single agent or in combination therapy. ${ }^{1}$ Central nervous system involvement (CNSi) is a rare complication of $\mathrm{CLL}^{2,3}$ and is reported in approximately $0.4 \%$ of patients. ${ }^{3}$ Therapeutic guidelines have not yet been established and prognosis for these patients is poor. ${ }^{4}$ Few cases of CLL with CNSi have been reported until now, and ibrutinib was successfully used due to its ability to penetrate the blood-brain barrier. $^{5}$

Here we report the measurements of therapeutic concentrations of venetoclax in cerebrospinal fluid (CSF) in CLL patients with CNSi, relapsed during ibrutinib therapy.

A 58-year old patient with trisomy 12, IGHV unmutated (VH4L) CLL relapsed with CNSi in September 2016. He had previously received six FCR courses, six RBendamustine cycles, and four R-DHAP courses. Magnetic resonance imaging (MRI) showed bilateral frontal periventricular lesions and meningeal involvement at L4-L5, both suggesting disease localization. Immunophenotyping on CSF showed lymphoid scatter cells $(90 \%)$ with atypical CLL phenotype [CD19+ $\left.\operatorname{sIgk}^{+}(\operatorname{dim}) \mathrm{CD}_{23}^{+/}(32 \%) \mathrm{CD}^{-} \mathrm{CD}_{20}^{-}\right]$(Table 1$)$. No evidence of extra-CNS involvement was documented. After response to ibrutinib and IT chemotherapy (cytarabine plus methotrexate) disease progressed in July 2017 (10 months after starting ibrutinib). MRI showed a more evident signal in the lumbosacral and medullary cone (Figure 1A). Immunophenotyping on CSF documented monoclonal B-lymphocyte population consistent with the diagnostic phase (Table 1). Computed tomography (CT) scan excluded systemic disease relapse.

Venetoclax was started together with IT chemotherapy (cytarabine $70 \mathrm{mg}$ plus methotrexate $15 \mathrm{mg}$ ) twice in the first week, weekly during ramp-up until the full dose of venetoclax was reached, and then monthly for 3 months (6 ITs during ramp-up and 3 thereafter). Physicochemical, morphological and flow cytometry on CSF were performed at each lumbar puncture. One month after venetoclax initiation, CSF clearance was gained and maintained at subsequent re-evaluations (Table 1). MRI response was documented in November 2017 (Figure 1B) and is still persistent (September 2018).

After three months of venetoclax full-dose therapy, in a condition of drug steady-state concentration in plasma, ${ }^{6}$ blood and CSF samples were taken 2 and 23 hours (h) after the daily oral dose of $400 \mathrm{mg}$ of the drug, respectively, in November and December 2017, after the patient had given written informed consent.

Venetoclax was quantified in plasma and CSF by liquid chromatography coupled to tandem mass spectrometry, after protein precipitation with acetonitrile. The mass spectrometer operated in multiple-reaction monitoring mode and measured the mass-charge ratio $(\mathrm{m} / \mathrm{z})$ reaction 868-321. The limit of quantitation in all specimens was $0.1 \mathrm{ng} / \mathrm{mL}$.

Venetoclax plasma concentrations, $\mathrm{Cmax}$ and $\mathrm{Cmin}, 2$ and $23 \mathrm{~h}$ after the oral dose, were 1.2 and $0.52 \mu \mathrm{g} / \mathrm{mL}$. At the same times, the drug distributed in the CNS reached concentrations in the CSF of 2.8 and $1.5 \mathrm{ng} / \mathrm{mL}$. The percentage of CSF concentration to plasma concentration was $0.1 \%$, corresponding to the free drug present as unbound fraction in plasma, which is the fraction of the drug which can be distributed into the CNS. ${ }^{8}$

The trough drug level of $1.5 \mathrm{ng} / \mathrm{mL}$ found in the CSF, which is the lowest concentration reached by the drug before the next dose, was close to the IC50 (1.9 nM, 1.6 $\mathrm{ng} / \mathrm{mL}$ ) established in vitro in a cultured CLL cell line exposed for $24 \mathrm{~h}$ to venetoclax. ${ }^{9}$ It is reasonable to suggest that this amount can effectively inhibit tumor growth at the CNS site, also considering that chronic treatment will induce continuous exposure of cancer cells to active venetoclax concentrations.

For the first time, we observed that the drug crossed

Table 1. Cerebrospinal fluid (CSF) analysis performed at the time of first central nervous system (CNS) (A) progression, (B) before venetoclax, and (C) after 4 months of venetoclax therapy.

\begin{tabular}{|c|c|c|c|}
\hline \multicolumn{2}{|r|}{ A. Before lbrutinib } & B. Before Venetoclax & C. After Venetoclax \\
\hline Visual inspection & Cloudy & Cloudy & Limpid \\
\hline Color & Colorless & Slightly xantrochomic & Colorless \\
\hline Glucose, mg/dL (normal ran & ge 40-70) & 4 & 86 \\
\hline Proteins, mg/dL (normal ra & ge 10-45) & 411 & 56 \\
\hline Erythrocytes & Absent & Absent & Absent \\
\hline Leukocytes, $\times 10^{9} / \mathrm{L}$ & 16 & 660 & $<1$ \\
\hline Morphological examination & Atypical lymphocytes & Atypical lymphocytes & No cells \\
\hline Flow cytometry & $\begin{array}{c}90 \% \mathrm{CD}^{1} 9^{+} \operatorname{sIgk}^{+}(\mathrm{dim}) \\
\mathrm{CD}^{2} 3^{+}(32 \%) \mathrm{CD}^{-} \mathrm{CD}^{-} 0^{-} \\
\text {B lymphocytes sIgk(dim)-restricted }\end{array}$ & $\begin{array}{c}\text { 70\%: } \\
\mathrm{CD}^{+}{ }^{+} \text {slgk }^{+}(\text {dim }) \mathrm{CD}_{2} 3^{+} \mathrm{CD}^{-} \\
\text {CD10-CD20 } \\
\text { B lymphocytes } \\
\text { slgk(dim)-restricted } \\
\text { 30\%: } \text { CD5 }^{+} \text {polyclonal } \\
\text { T lymphocytes }\end{array}$ & $\begin{array}{c}34 \text { cells, all } \mathrm{CD}^{+} \text {polyclonal } \\
\text { T lymphocytes }\end{array}$ \\
\hline
\end{tabular}

Physico-chemical, morphological and flow cytometry parameters at the time of progression were all consistent with central nervous system localization of pathological monoclonal B lymphocytes. During venetoclax therapy, hypoglycorrhachia, hyperprotidorrhachia and pathological B lymphocytes were no longer detectable. CD: cluster of differentiation. 
A

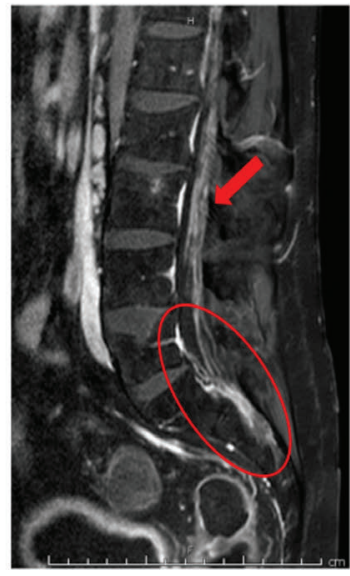

B
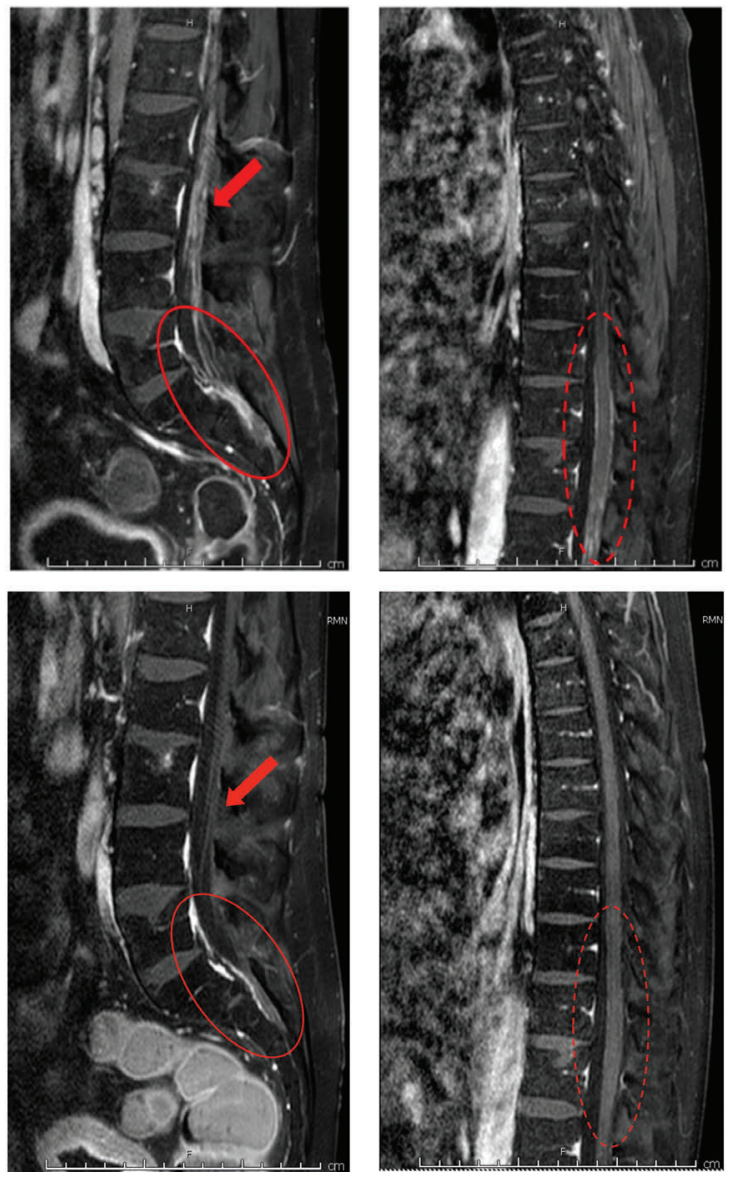

Figure 1. Spinal magnetic resonance imaging (MRI) performed at the time of progression after ibrutinib therapy and after 4 months of venetoclax therapy. The figure shows the lumbar (left boxes) and thoracic (right boxes) spine in T1-weighted Spectral Presaturation with Inversion Recovery sequences after gadolinium injection. (A) Spinal MRI at the time of progression after ibrutinib: contrast-enhanced lesions involve the lumbosacral roots (red circle), conus medullaris (red arrow) and the dural sac surrounding the dorsal spinal cord (dashed red circle). (B) Spinal MRI after 4 months of venetoclax: the lesions previously described are no longer detectable.

the blood-brain barrier with a concentration close to the IC50 in CLL, and we report a potential efficacy of venetoclax combined with IT treatment in CLL with CNSi. In fact, a contribution of IT therapy to CNS disease clearance is likely, particularly in the early phase, where an additive effect of venetoclax can be hypothesized; moreover, the drug played a major role in the long-term response.
The blood-brain barrier penetration of venetoclax, together with its broad spectrum of action in hematologic malignancies, may lead the way to new treatment options in CNS localizations.

Gianluigi Reda, ${ }^{1{ }^{*}}$ Ramona Cassin, ${ }^{1 *}$ Gabriela Dovrtelova, ${ }^{2,3}$ Cristina Matteo, ${ }^{2}$ Juri Giannotta, ${ }^{1}$ Maurizio D'Incalci, ${ }^{2}$ Agostino Cortelezzi' and Massimo Zucchetti ${ }^{2}$

'Hematology Unit, IRCCS Ca' Granda Ospedale Maggiore Policlinico of Milan, Italy; ${ }^{2}$ Department of Oncology, Istituto di Ricerche Farmacologiche Mario Negri IRCCS, Italy and ${ }^{3}$ Department of Pharmacology, Faculty of Medicine, Masaryk University and International Clinical Research Center, St. Anne's University Hospital Brno, Czech Republic

${ }^{*} G R$ and $R C$ contributed equally to this work.

Correspondence: GIANLUIGI REDA

gianluigi.reda@policlinico.mi.it

doi:10.3324/haematol.2018.213157

Information on authorship, contributions, and financial \& other disclosures was provided by the authors and is available with the online version of this article at www. haematologica.org.

\section{References}

1. Roberts AW, Davids MS, Pagel JM, et al. Targeting BCL2 with venetoclax in relapsed chronic lymphocytic leukemia. $N$ Engl J Med. 2016;374(4):311-322.

2. Ratterman M, Kruczek K, Sulo S, Shanafelt TD, Kay NE, Nabhan C. Extramedullary chronic lymphocytic leukemia: systematic analysis of cases reported between 1975 and 2012. Leuk Res. 2014;38(3):299303.

3. Strati P, Uhm JH, Kaufmann TJ, et al. Prevalence and characteristics of central nervous system involvement by chronic lymphocytic leukemia. Haematologica. 2016;101(4):458-465.

4. Moazzam AA, Drappatz J, Kim RY, Kesari S. Chronic lymphocytic leukemia with central nervous system involvement: report of two cases with a comprehensive literature review. J Neurooncol. 2012; 106(1):185-200

5. Wanquet A, Birsen R, Lemal R, Hunault M, Leblond V, AurranSchleinitz T. Ibrutinib responsive central nervous system involvement in chronic lymphocytic leukemia. Blood. 2016;127(19):23562358.

6. Salem AH, Agarwal SK, Dunbar M, Enschede SL, Humerickhouse RA, Wong SL. Pharmacokinetics of venetoclax, a novel BCL-2 inhibitor, in patients with relapsed or refractory chronic lymphocytic leukemia or non-Hodgkin lymphoma. J Clin Pharmacol. 2017; 57(4):484-492.

7. Salem AH, Hu B, Freise KJ, Agarwal SK, Sidhu DS, Wong SL. Evaluation of the pharmacokinetic interaction between venetoclax, a selective BCL-2 inhibitor, and warfarin in healthy volunteers. Clin Drug Investig. 2017;37(3):303-309.

8. Emami Riedmaier A, Lindley DJ, Hall JA, et al. Mechanistic physiologically based pharmacokinetic modeling of the dissolution and food effect of a biopharmaceutics classification system IV compound-The venetoclax story. J Pharm Sci. 2018;107(1):495-502.

9. Anderson MA, Deng J, Seymour JF, et al. The BCL2 selective inhibitor venetoclax induces rapid onset apoptosis of CLL cells in patients via a TP53-independent mechanism. Blood. 2016;1 27(25):3215-3224. 